\title{
Stability of curvature perturbation with new covariant form for energy-momentum transfer in dark sector
}

\author{
Cheng-Yi Sun ${ }^{* 1}$, Yu Song ${ }^{1}$ and Rui-Hong Yue ${ }^{2}$ \\ ${ }^{1}$ Institute of Modern Physics, Northwest University, Xian 710069, P.R. China. \\ ${ }^{2}$ Faculty of Science, Ningbo University, Ningbo 315211, P.R. China.
}

December 5, 2018

\begin{abstract}
It was found that the model with interaction between cold dark matter (CDM) and dark energy (DE) proportional to the energy density of CDM $\rho_{m}$ and constant equation of state of DE $w_{d}$ suffered from instabilities of the density perturbations on the supper-Hubble scales. Here we suggest a new covariant model for the energy-momentum transfer between CDM and DE. Then using the covariant model, we analyze the evolution of density perturbations on the supper-Hubble scale. We find that the instabilities can be avoided in the model with constant $w_{d}$ and interaction proportional to $\rho_{m}$. Furthermore, we analyze the dominant non-adiabatic mode in the radiation era and find that the mode grows regularly.
\end{abstract}

\section{Introduction}

We are convinced by the increasing observations [1, 2, 3] that the present universe is dominated by the so called dark energy (DE), which accounts for $\simeq 70 \%$ of the critical mass density and has been pushing the universe into accelerated expansion [4, 5]. And the other main component in the universe is cold dark matter (CDM), which accounts for $\simeq 30 \%$ of the critical mass density and behaves as the pressureless dust. Then it is natural for us to consider that the two dark components might interact mutually. And furthermore it is found that an appropriate interaction can help to alleviate the coincidence problem [6, 7], namely why DE and CDM are comparable in size exactly today [5]. Different interacting models of dark energy have been investigated intensively [8, 9].

*cysun@mailis.gucas.ac.cn; ddscy@163.com 
Usually, in the literature, the model with interaction between DE and CDM is described by the two continuity equations

$$
\begin{aligned}
\dot{\rho}_{m}+3 H \rho_{m} & =Q, \\
\dot{\rho}_{d}+3 H\left(1+w_{d}\right) \rho_{d} & =-Q,
\end{aligned}
$$

where $Q$ denotes the phenomenological interaction term between DE and CDM; $\rho_{m}$ and $\rho_{d}$ are the energy densities of CDM and DE respectively; $w_{d} \equiv p_{d} / \rho_{d}$ is the equation of state parameter of $\mathrm{DE} ; p_{d}$ is the pressure density of $\mathrm{DE} ; H \equiv \dot{a} / a$ is the Hubble parameter; $a(t)$ is the scale factor in the Friedmann-Robertson-Walker (FRW) metric; a dot denotes the derivative with respect to the cosmic time $t$. In the note we do not allow the phantom case $w_{d}<-1$. Owing to the lack of the knowledge of micro-origin of the interaction, usually the interaction term is parameterized in a simple form as [6]

$$
Q=3 H\left(\alpha \rho_{m}+\beta \rho_{d}\right)
$$

where $\alpha$ and $\beta$ are positive constants. The interaction term $Q$ would influence not only the background dynamics of the universe, but also the growth of the perturbations of the cosmological fluids.

Recently, in Ref.[10], by modeling DE as a fluid with constant $w_{d}>-1$, the authors investigated the evolution of the linear density perturbations and have shown that the combination of constant $w_{d}$ and the simple interaction form $Q$ given in Eq.(3) leads to an instability: the curvature perturbation on the super-Hubble scales blows up in the early universe [10]. The explicit models investigated in Ref.[10] included the two cases of $\beta=0$ and $\alpha=\beta$ in Eq.(33). Further more, in [12], it is concluded that the perturbations in the dark energy become unstable for any model with constant $w_{d}>-1$ and non-zero $\alpha$, no matter how small the parameter $\alpha$ is made. In [11, 12, 13], the case of $\alpha=0$ was surveyed and it was found that the instability can be avoided if $\beta$ is made small enough. In [14], by modeling DE as a quintessence field, the author found that the instability can also be avoided even for the interaction $Q$ proportional to $\rho_{m}$.

Then it seems that the model with constant $w_{d}$ and non-zero $\alpha$ in Eq.(3) is ruled out as a viable interacting model. However, in the note we try to show that the evolution of the density perturbations becomes regular even in the case of $\beta=0$ in Eq.(3) if we adopt a new covariant form for energy-momentum transfer between DE and CDM.

The remain part of the note is organized as follows. Firstly, we display our new covariant model for interaction in the dark sector. Secondly, by assuming the universe filled only by DE and CDM, we investigate the evolution of the density perturbations and show that instability can be avoided. Thirdly, by considering the effects of the radiation (photons and neutrinos) and baryons, we survey the dominant non-adiabatic mode in the radiation era and show that no non-adiabatic mode blows up. Finally, conclusions and discussions are given. 


\section{A Covariant Model For Dark-Sector Interaction}

The conservation laws (11) and (2) work well in describing the background evolution of the universe. But in order to study the evolution of the density perturbation, we need a covariant form for the energy-momentum transfer between DE and CDM which holds in an inhomogeneous universe and reduces to Eqs.(11) and (2) in a homogeneous FRW universe. Usually, the covariant form for energy-momentum transfer is taken to be [15, 10]

$$
\nabla_{\nu} T_{A}^{\mu \nu}=Q_{A}^{\mu}, \quad \sum_{A} Q_{A}^{\mu}=0
$$

where $A=m, d$ to denote CDM and DE respectively. In Ref.[10], it is assumed that

$$
Q_{m}^{\mu}=-Q_{d}^{\mu}=Q u_{m}^{\mu}
$$

where $u_{m}^{\mu}$ is the four velocity of CDM. The conclusions in [10] are based on the above ansatz.

However, we find that there may exist the other natural covariant form for the energymomentum transfer. Let us show it. In the note, we take the interaction $Q$ to be

$$
Q=3 \alpha H \rho_{m},
$$

This is just the case of $\beta=0$ in Eq.(3). Then we can rewrite Eqs.(11) and (2) as

$$
\begin{array}{r}
\dot{\rho}_{m}+3 H\left(\rho_{m}+p_{m}^{\text {eff }}\right)=0 \\
\dot{\rho}_{d}+3 H\left(\rho_{d}+p_{d}^{\text {eff }}\right)=0
\end{array}
$$

where

$$
\begin{aligned}
& p_{m}^{\mathrm{eff}} \equiv-\alpha \rho_{m} \\
& p_{d}^{\mathrm{eff}} \equiv p_{d}+\alpha \rho_{m}
\end{aligned}
$$

Motivated by these equations, we may define the effective energy-momentum tensors of CDM and DE respectively as

$$
\begin{aligned}
& T_{\mathrm{em}}^{\mu \nu}=\rho_{m} u_{m}^{\mu} u_{m}^{\nu}+p_{m}^{\mathrm{eff}}\left(u_{m}^{\mu} u_{m}^{\nu}+g^{\mu \nu}\right) \\
& T_{\mathrm{ed}}^{\mu \nu}=\rho_{d} u_{d}^{\mu} u_{d}^{\nu}+p_{d}^{\mathrm{eff}}\left(u_{d}^{\mu} u_{d}^{\nu}+g^{\mu \nu}\right)
\end{aligned}
$$

where $u_{m}^{\mu}$ and $u_{d}^{\mu}$ are the four velocities of CDM and DE respectively. And the two effective energy-momentum tensors are conserved respectively

$$
T_{\mathrm{em} ; \mu}^{\mu \nu}=T_{\mathrm{ed} ; \mu}^{\mu \nu}=0
$$


It can be easily checked that Eqs.(11) and (21) can be reduced from Eq.(13) in the background FRW universe. The corresponding Einstein equations should be

$$
G^{\mu \nu}=8 \pi G\left(T_{\mathrm{em}}^{\mu \nu}+T_{\mathrm{ed}}^{\mu \nu}\right) .
$$

Now we can survey the evolution of the curvature perturbation by expanding Eqs.(13) and (14) to the first order of the density perturbations.

The covariant model given in the last paragraph is very different from the one defined in Eq.(4), although both of them give the same background evolution of the universe. In the model in Eq.(4), the perturbations of the interaction effect the evolution of the density perturbations via the continuity equations and do not appear in the Einstein equations explicitly, while in the model defined in the last paragraph, the perturbations of the interaction appears explicitly both in the continuity equations and in the Einstein equations.

\section{Evolution of Density Perturbations}

In the section, we apply the covariant model defined in Eqs.(13) and (14) to study the evolution of the density perturbations in the model with $Q$ given in Eq.(6) and constant $w_{d}$. For simplicity, we consider a flat FRW universe filled only by DE and CDM. The perturbed FRW metric in the conformal Newtonian gauge is given by

$$
d s^{2}=a^{2}(\tau)\left[-(1+2 \phi) d \tau^{2}+(1-2 \psi) d \mathbf{x}^{2}\right],
$$

where $\phi$ and $\psi$ denote the scalar perturbations. The corresponding Friedmann equation can be rewritten as

$$
\mathcal{H}^{2} \equiv\left(\frac{a^{\prime}}{a}\right)^{2}=\frac{8 \pi G}{3}\left(\rho_{m}+\rho_{d}\right) a^{2} .
$$

Hereafter, primes denote the derivatives with respect to the conformal time $\tau$. With Eq.(6) and constant $w_{d}$, the background continuity equations (11) and (2) can be solved exactly:

$$
\begin{aligned}
\rho_{m} & =\rho_{m 0} a^{-3(1-\alpha)}, \\
\rho_{d} & =\rho_{d 0} a^{-3\left(1+w_{d}\right)}+\left(\frac{\alpha}{\alpha+w_{d}}\right) \rho_{m 0} a^{-3}\left(a^{-3 w_{d}}-a^{3 \alpha}\right) .
\end{aligned}
$$

Hereafter, the subscript 0 denotes the present value of the corresponding parameter and $a_{0}=1$.

\subsection{Evolving Equations of Density Perturbations}

When the perturbed metric in Eq.(15) is considered, the four velocities of CDM and DE are

$$
u_{m}^{\mu}=a^{-1}\left(1-\phi, \partial_{i} v_{m}\right), \quad u_{d}^{\mu}=a^{-1}\left(1-\phi, \partial_{i} v_{d}\right),
$$


where $v_{m}$ and $v_{d}$ are the peculiar velocity potentials of CDM and DE respectively. Usually, we define the volume expansion rates of CDM and DE (in Fourier space) respectively as

$$
\theta_{m}=-k^{2} v_{m}, \quad \theta_{d}=-k^{2} v_{d}
$$

We use $\delta \rho_{m}, \delta \rho_{d}, \delta p_{d}, \delta p_{m}^{\text {eff }}$ and $\delta p_{d}^{\text {eff }}$ to denote the first-order perturbations of the corresponding parameters. Then we can introduce the dimensionless factional density perturbations of CDM and DE as

$$
\delta_{m}=\frac{\delta \rho_{m}}{\rho_{m}}, \quad \delta_{d}=\frac{\delta \rho_{d}}{\rho_{d}} .
$$

The curvature perturbation on the constant- $\rho_{A}(A=m, d)$ surface and the total curvature perturbation on the constant- $\rho\left(\rho=\rho_{m}+\rho_{d}\right)$ surface are defined respectively as

$$
\zeta_{A}=-\psi-\mathcal{H} \frac{\delta \rho_{A}}{\rho_{A}^{\prime}}, \quad \zeta=-\psi-\mathcal{H} \frac{\delta \rho_{m}+\delta \rho_{d}}{\rho_{m}^{\prime}+\rho_{d}^{\prime}} .
$$

From Eq.(9), we have

$$
\delta p_{m}^{\mathrm{eff}}=-\alpha \rho_{m} \delta_{m}
$$

And from Eq.(10), we have

$$
\delta p_{d}^{\mathrm{eff}}=\delta p_{d}+\alpha \rho_{m} \delta_{m}
$$

Here, following the analysis in Ref.[10], we take

$$
\delta p_{d}=\delta \rho_{d}+\left(1-w_{d}\right)\left[3 \mathcal{H}\left(1+w_{d}+\alpha \frac{\rho_{m}}{\rho_{d}}\right) \rho_{d}\right] \frac{\theta_{d}}{k^{2}} .
$$

Then from Eq.(13), we can get the evolving equations of the density and velocity perturbations of CDM and DE as

$$
\begin{array}{r}
\delta_{m}^{\prime}+(1-\alpha) \theta_{m}-3(1-\alpha) \psi^{\prime}=0 \\
\theta_{m}^{\prime}+\mathcal{H}(1+3 \alpha) \theta_{m}-k^{2} \phi+k^{2} \frac{\alpha}{1-\alpha} \delta_{m}=0
\end{array}
$$

and

$$
\begin{aligned}
& \delta_{d}^{\prime}+3 \mathcal{H}\left(1-w_{d}-\alpha \frac{\rho_{m}}{\rho_{d}}\right) \delta_{d}+9 \mathcal{H}^{2}\left(1-w_{d}\right)\left(1+w_{d}+\alpha \frac{\rho_{m}}{\rho_{d}}\right) \frac{\theta_{d}}{k^{2}} \\
&+3 \alpha \mathcal{H} \frac{\rho_{m}}{\rho_{d}} \delta_{m}+\left(1+w_{d}+\alpha \frac{\rho_{m}}{\rho_{d}}\right)\left(\theta_{d}-3 \psi^{\prime}\right)=0 \\
& \theta_{d}^{\prime}-2 \mathcal{H} \theta_{d}-3 \mathcal{H} \frac{\alpha(1-\alpha) \frac{\rho_{m}}{\rho_{d}}}{1+w_{d}+\alpha \frac{\rho_{m}}{\rho_{d}}} \theta_{d}-k^{2} \phi-k^{2} \frac{\alpha \frac{\rho_{m}}{\rho_{d}}}{1+w_{d}+\alpha \frac{\rho_{m}}{\rho_{d}}} \delta_{m}-k^{2} \frac{\delta_{d}}{1+w_{d}+\alpha \frac{\rho_{m}}{\rho_{d}}}=0
\end{aligned}
$$


In the conformal Newtonian gauge, the first-order perturbed Einstein equations Eq.(14) give us 16

$$
\begin{aligned}
& 3 \mathcal{H} \psi^{\prime}+k^{2} \psi+3 \mathcal{H}^{2} \phi=-4 \pi G a^{2}\left(\delta_{m} \rho_{m}+\delta_{d} \rho_{d}\right), \\
& k^{2} \psi^{\prime}+k^{2} \mathcal{H} \phi=4 \pi G a^{2}\left[(1-\alpha) \rho_{m} \theta_{m}+\left(1+w_{d}+\alpha \frac{\rho_{m}}{\rho_{d}}\right) \rho_{d} \theta_{d}\right] \\
& \psi^{\prime \prime}+\mathcal{H}\left(2 \psi^{\prime}+\phi^{\prime}\right)+\left(2 \frac{a^{\prime \prime}}{a}-\mathcal{H}^{2}\right) \phi+\frac{k^{2}}{3}(\psi-\phi)=4 \pi G a^{2}\left(\delta p_{m}^{\mathrm{eff}}+\delta p_{d}^{\mathrm{eff}}\right) \\
& \psi-\phi=0 .
\end{aligned}
$$

Only two of the above equations are independent. Choosing two of them (e.g. Eqs.(30) and (33)), and using Eqs.(26), (27), (28) and (29) together, we can solve these evolving equations numerically, if the initial conditions are given.

\subsection{Adiabatic Initial Conditions}

In the early universe, $a \ll 1$, Eqs.(17) and (18) indicate

$$
\frac{\rho_{m}}{\rho_{d}} \rightarrow-\frac{w_{d}+\alpha}{\alpha}
$$

and then, from Eq.(16), we have

$$
\mathcal{H}=\frac{2}{1-3 \alpha} \tau^{-1}, \quad \tau=\frac{2}{(1-3 \alpha) H_{0}} \sqrt{\frac{w_{d}+\alpha}{w_{d} \Omega_{m 0}}} a^{\frac{1}{2}(1-3 \alpha)},
$$

where

$$
\Omega_{m 0} \equiv \frac{8 \pi G \rho_{m 0}}{3 H_{0}^{2}}
$$

Here we adopt the adiabatic initial conditions to study the evolution of the density perturbations on the super-Hubble scales $(k \ll a H)$. To the lowest order in $k \tau$, we can set the adiabatic conditions as

$$
\begin{aligned}
\phi & =\psi=A_{\phi}=\text { Const., } \\
\delta_{m} & =\delta_{d}=-\frac{2 w_{d}}{w_{d}+\alpha} A_{\phi}, \\
\theta_{m} & =\frac{\left(w_{d}+\alpha+\alpha w_{d}-\alpha^{2}\right)(1-3 \alpha)}{3\left(1-\alpha^{2}\right)\left(w_{d}+\alpha\right)} k^{2} \tau A_{\phi}, \\
\theta_{d} & =\frac{\left[2 w_{d}^{2}-(1-\alpha)\left(w_{d}-\alpha\right)\right](1-3 \alpha)}{3(1-\alpha)\left(w_{d}+\alpha\right)\left(2 w_{d}+\alpha-1\right)} k^{2} \tau A_{\phi} .
\end{aligned}
$$




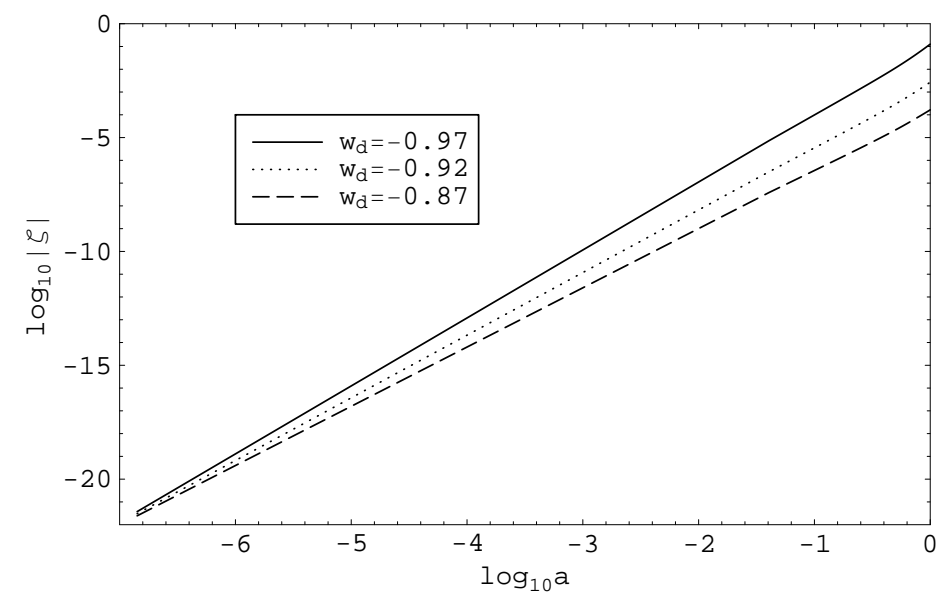

Fig. 1: $\log _{10}|\zeta|$ versus $\log _{10} a$ in the interacting model for fixed $\Omega_{m 0}=0.3, h=0.67, k=$ $1.5 \times 10^{-4} \mathrm{Mpc}^{-1}, \alpha=10^{-3}$ and different $w_{d}$.

\subsection{Evolution of Curvature Perturbation}

Now using the initial conditions given in the last subsection, we can solve the evolving equations (26)-(30) and (33) numerically to obtain the evolution of the density perturbation and then get the evolution of the curvature perturbation $\zeta$ by using Eq.(22). We have displayed the results in Fig [1 and Fig.2. In the two figures, we have fixed the parameters as $\Omega_{m 0}=0.3, k=1.5 \times 10^{-4} \mathrm{Mpc}^{-1}$, $H_{0}=100 h \mathrm{~km} \mathrm{sec}^{-1} \mathrm{Mpc}^{-1}$ and $h=0.67$. And we have taken $A_{\phi}=10^{-25}$ and the initial values of $a$ to be $a=10^{-11}$. In Fig, 1, we show the evolutions of $\log _{10}|\zeta|$ for fixed $\alpha=1 \times 10^{-3}$ and different $w_{d}$. And in Fig, 2 we show the evolutions of $\log _{10}|\zeta|$ for fixed $w_{d}=-0.94$ and different $\alpha$. The evolutions displayed in Fig,1 and Fig,2 manifest the standard power-law growth and no instabilities are present, which are similar the results in [14].

\section{Dominant Non-Adiabatic Mode}

In the last section, we have shown that in our new covariant model, instabilities on the superHubble scale can be avoided. But the conclusion is obtained by assuming that the universe is filled only by CDM and DE. In this section, we discuss the dominant non-adiabatic mode deep in the radiation-dominated era by including the components of photons, neutrinos and baryons in the universe. If the dominant non-adiabatic mode evolves regular in the radiation era, we believe the instabilities can also be avoided even when the components of radiations and baryons are involved. 


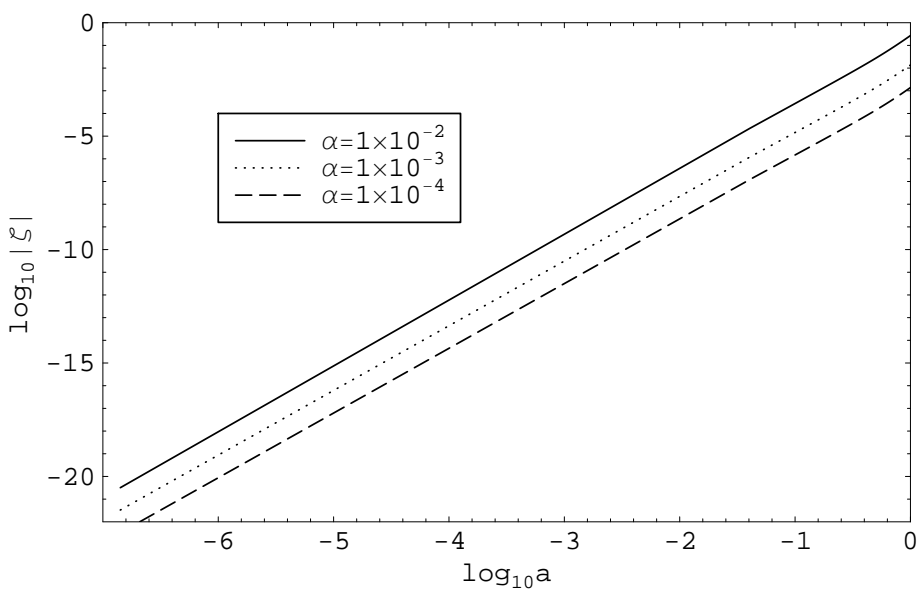

Fig. 2: $\log _{10}|\zeta|$ versus $\log _{10} a$ in the interacting model for fixed $\Omega_{m 0}=0.3, h=0.67, k=$ $1.5 \times 10^{-4} \mathrm{Mpc}^{-1}, w_{d}=-0.94$ and different $\alpha$.

The $A$-fluid energy-momentum tensor including perturbations is taken to be

$$
T_{A}^{\mu \nu}=\left(\rho_{A}+p_{A}\right) u_{A}^{\mu} u_{A}^{\nu}+p_{A} g^{\mu \nu}+\pi_{A}^{\mu \nu},
$$

where $u_{A}^{\mu}$ is the four velocity

$$
u_{A}^{\mu}=a^{-1}\left(1-\phi, \partial_{i} v_{A}\right)
$$

We have allowed an anisotropic shear perturbation $\pi_{A}^{\mu \nu}$, and $A=b, \gamma, \nu$ to denote the corresponding parameters of baryons, photons and neutrinos. We take $\pi_{b}^{\mu \nu}=\pi_{\gamma}^{\mu \nu}=0$ and

$$
\pi_{\nu}^{0 \mu}=0, \quad \pi_{\nu}^{i j}=a^{-2}\left(\partial_{i} \partial_{j}-\frac{1}{3} \delta^{i j}\right) \pi_{\nu}
$$

The fluids of CDM and DE are described by the effective energy-momentum tensors defined in Eqs.(11) and (12) respectively.

Early in the radiation era, the Friedmann equation reads

$$
\mathcal{H}^{2} a^{-2}=\frac{8 \pi G}{3}\left(\rho_{\gamma}+\rho_{\nu}\right)=\frac{8 \pi G}{3} \rho_{r 0} a^{-4} .
$$

Then we have

$$
a=\sqrt{\Omega_{r 0}} H_{0} \tau, \quad \mathcal{H}=\tau^{-1}, \quad \Omega_{r 0} \equiv \frac{8 \pi G \rho_{r 0}}{3 H_{0}^{2}} .
$$


In the radiation era, the perturbed Einstein equations give us that

$$
\begin{aligned}
& 3 \tau^{-1} \psi^{\prime}+k^{2} \psi+3 \tau^{-2} \phi=-4 \pi G a^{2}\left(\delta \rho_{m}+\delta \rho_{d}+\sum_{A} \delta \rho_{A}\right), \\
& k^{2}\left(\psi^{\prime}+\tau^{-1} \phi\right)=4 \pi G a^{2}\left[\left(\rho_{m}+p_{m}^{\mathrm{eff}}\right) \theta_{A}+\left(\rho_{d}+p_{d}^{\mathrm{eff}}\right) \theta_{A}+\sum_{A}\left(\rho_{A}+p_{A}\right) \theta_{A}\right], \\
& \psi^{\prime \prime}+2 \tau^{-1} \psi^{\prime}+\tau^{-1} \phi^{\prime}-\tau^{-2} \phi+\frac{k^{2}}{3}(\psi-\phi)=4 \pi G a^{2}\left(\delta p_{m}^{\mathrm{eff}}+\delta p_{d}^{\mathrm{eff}}+\sum_{A} \delta p_{A}\right), \\
& \psi-\phi=8 \pi G \pi_{\nu},
\end{aligned}
$$

where $A$ runs over $b, \gamma$, and $\nu$.

For CDM and DE, the perturbed continuity equations are given by Eqs.(26) $-(29)$. For baryons, the perturbed continuity equations (in Fourier space) are [10]

$$
\begin{aligned}
& \delta_{b}^{\prime}=-\theta_{b}+3 \psi^{\prime}, \\
& \theta_{b}^{\prime}=-\mathcal{H} \theta_{b}+k^{2} \phi
\end{aligned}
$$

and for photons $[10$

$$
\begin{aligned}
& \delta_{\gamma}^{\prime}=-\frac{4}{3} \theta_{\gamma}+4 \psi^{\prime}, \\
& \theta_{\gamma}^{\prime}=\frac{1}{4} k^{2} \delta_{\gamma}+k^{2} \phi
\end{aligned}
$$

and for neutrinos [10]

$$
\begin{aligned}
\delta_{\nu}^{\prime} & =-\frac{4}{3} \theta_{\nu}+4 \psi^{\prime}, \\
\theta_{\nu}^{\prime} & =\frac{1}{4} k^{2} \delta_{\nu}+k^{2} \phi-k^{2} \sigma_{\nu}, \\
\sigma_{\nu}^{\prime} & =\frac{4}{15} \theta_{\nu},
\end{aligned}
$$

where $\theta_{A}=-k^{2} v_{A}$ and $\sigma_{\nu} \equiv 2 k^{2} \pi_{\nu} /\left[3 a^{2}\left(\rho_{\nu}+p_{\nu}\right)\right]$

In order to find the dominant non-adiabatic mode, we assume a leading-order power law for perturbations [10]

$$
\psi=A_{\psi}(k \tau)^{n_{\psi}}, \phi=A_{\phi}(k \tau)^{n_{\phi}}, \delta_{A}=B_{A}(k \tau)^{n_{A}}, \theta_{A}=C_{A}(k \tau)^{s_{A}}, \sigma_{\nu}=D_{\nu}(k \tau)^{n_{\sigma}} .
$$


Here the subscript $A=c, d, b, \gamma$, and $\nu$ denotes the corresponding parameter of CDM, DE, baryons, photons and neutrinos respectively. To the leading order in $k \tau$, the equations (26)-(29) and (44)(54) may be solved, in terms of $\psi$ :

$$
\begin{aligned}
\phi & =J \psi, \quad J=1-\frac{16 R_{\nu}}{5\left(n_{\psi}+2\right)\left(n_{\psi}+1\right)+8 R_{\nu}}, \\
\delta_{\gamma} & =\delta_{\nu}=4 \psi, \quad \theta_{\gamma}=\theta_{\nu}=\frac{J+1}{n_{\psi}+1} k^{2} \tau \psi, \\
\delta_{b} & =3 \psi, \quad \theta_{b}=\frac{J}{n_{\psi}+2} k^{2} \tau \psi, \\
\delta_{m} & =3(1-\alpha) \psi, \quad \theta_{m}=\frac{J-3 \alpha}{n_{\psi}+2+3 \alpha} k^{2} \tau \psi, \\
\delta_{d} & =\frac{2 \Omega_{r 0}^{(1-3 \alpha) / 2}}{\alpha \Omega_{m 0} H_{0}^{1+3 \alpha}}\left(w_{d}+\alpha\right)\left(n_{\psi}+J+2\right) \frac{\psi}{\tau^{1+3 \alpha}}, \\
\theta_{d} & =-\frac{n_{\psi}+2}{9\left(1-w_{d}\right)(1-\alpha)} k^{2} \tau \delta_{d},
\end{aligned}
$$

where $R_{\nu} \equiv \rho_{\nu} /\left(\rho_{\gamma}+\rho_{\nu}\right)$ and

$$
n_{\psi}=\frac{-3 w_{d} \pm \sqrt{9 w_{d}^{2}+12 w_{d}-20}}{2}
$$

Eq.(60) indicates that the modes are regular (i.e. well behaved as $k \tau \rightarrow 0$ ) provided

$$
\operatorname{Re}\left[n_{\psi}\right] \geq 1+3 \alpha .
$$

For $w_{d} \sim-1$, this leads to

$$
-\frac{3}{2} w_{d} \geq 1+3 \alpha \Rightarrow \alpha \lesssim \frac{1}{6}
$$

Correspondingly, the total curvature perturbation $\zeta$ is defined as

$$
\zeta=-\psi-\mathcal{H} \frac{\sum_{A} \delta \rho_{A}}{\sum_{A} \rho_{A}^{\prime}}
$$

where $A$ runs over $m, d, \gamma, \nu$ and $b$. Then $\zeta$ can be expressed in terms of $\psi$ as

$$
\zeta=-\frac{1}{2}\left(n_{\psi}+J+2\right) \psi .
$$

For $w_{d} \sim-1, n_{\psi}$ is a complex number and

$$
\operatorname{Re}\left[n_{\psi}\right] \sim \frac{3}{2}
$$

So the dominant non-adiabatic mode grows in a regular power law and no instabilities are present. 


\section{Conclusions and Discussions}

In the note, we have suggested a new covariant model for dark-sector interaction to avoid the instabilities of the curvature perturbation on the supper-Hubble scale. By using the covariant model and choosing $Q=3 \alpha H \rho_{m}$ and constant $w_{d}$, we analyze the evolution of density perturbations in a universe filled only by CDM and DE and find that the instabilities shown in [10] can be avoided in our covariant model. Further more, we analyze the dominant non-adiabatic mode early in the radiation era, and find the non-adiabatic mode evolves regularly. So we believe that in our covariant model, the instabilities can also be avoided even in the universe filled by radiation, matter and DE.

Actually, it is not very surprising for the instabilities to be avoided in our covariant model. We know, from Eqs.(11)-(14), that the two mutually-interacting dark fluids can be described respectively by two effective energy-momentum tensors which are conserved separately. This means the interacting model can be taken as a non-interacting model effectively, while no instabilities are present in non-interacting models. So we expect the instabilities can be avoided in our covariant model.

\section{Acknowledgments}

This work has been supported in part by the Research Fund for the Doctoral Program of Higher Education of China under Grant No. 20106101120023, the National Natural Science Foundation of China under Grant No. 10875060, and the Natural Science Foundation of the Northwest University of China under Grant No. 09NW27.

\section{References}

[1] A. G. Riess et al., Astron. J. 116, 1009 (1998), astro-ph/9805201]; S. Perlmutter et al., Astrophys. J. 517, 565 (1999), astro-ph/9812133].

[2] D. N. Spergel et al., Astrophys. J. Suppl. 148, 175 (2003), astro-ph/0302209]; D. N. Spergel et al., Astrophys. J. Suppl. 170, 377 (2007), astro-ph/0603449].

[3] M. Tegmark et al., Phys. Rev. D 69, 103501 (2004), astro-ph/0310723; K. Abazajian et al., Astron. J. 128, 502 (2004), astro-ph/0403325]; K. Abazajian et al., Astron. J. 129, 1755 (2005), astro-ph/0410239.

[4] S. Weinberg, Rev. Mod. Phys. 61, 1 (1989); 
[5] V. Sahni and A. A. Starobinsky, Int. J. Mod. Phys. D 9, 373 (2000), astro-ph/9904398; S. M. Carroll, Living Rev. Rel. 4, 1 (2001), astro-ph/0004075]; P. J. E. Peebles and B. Ratra, Rev. Mod. Phys. 75, 559 (2003), astro-ph/0207347]; T. Padmanabhan, Phys. Rept. 380, 235 (2003), [hep-th/0212290]; E. J. Copeland, M. Sami and S. Tsujikawa, Int. J. Mod. Phys. D 15, 1753 (2006), (hep-th/0603057); R. Bousso, Gen. Rel. Grav. 40, 607 (2008), arXiv:0708.4231[hep-th].

[6] L. P. Chimento, Journal of Mathematical Physics 38, 2565 (1997), arXiv:physics/9702029]; J. D. Barrow and T. Clifton, Phys. Rev. D 73, 103520 (2006), [gr-qc/0604063].

[7] L. Amendola, Phys. Rev. D 62, 043511 (2000), arXiv:astro-ph/9908023; L. P. Chimento, A. S. Jakubi, D. Pavon and W. Zimdahl, Phys. Rev. D 67, 083513 (2003), arXiv:astro-ph/0303145; R. G. Cai and A. Wang, JCAP 0503, 002 (2005), |arXiv:hep-th/0411025|; G. Olivares, F. Atrio-Barandela and D. Pavon, Phys. Rev. D 74, 043521 (2006), |arXiv:astro-ph/0607604|.

[8] L. P. Chimento, Phys. Rev. D 81, 043525 (2010), arXiv:0911.5687[astro-ph.CO]; J. H. He and B. Wang, JCAP 0806, 010 (2008), arXiv:0801.4233[astro-ph]; M. Szydlowski, T. Stachowiak and R. Wojtak, Phys. Rev. D 73, 063516 (2006), arXiv:astro-ph/0511650; L. P. Chimento, M. Forte and G. M. Kremer, Gen. Rel. Grav. 41, 1125 (2009), arXiv:0711.2646[astro-ph]; R. Bean, E. E. Flanagan, I. Laszlo and M. Trodden, Phys. Rev. D 78, 123514 (2008), arXiv:0808.1105[astro-ph]; C. Feng, B.Wang, Y. Gong and R. K. Su, JCAP 0709, 005 (2007), arXiv:0706.4033[astro-ph]; L. Zhang, J. Cui, J. Zhang and X. Zhang, Int. J. Mod. Phys. D 19, 21 (2010), arXiv:0911.2838[astro-ph.CO].

[9] S. Micheletti, E. Abdalla and B. Wang, Phys. Rev. D 79, 123506 (2009), arXiv:0902.0318[grqc]; S. Chen, B. Wang and J. Jing, Phys. Rev. D 78, 123503 (2008), arXiv:0808.3482[gr-qc]; H. Wei and R. G. Cai, Eur. Phys. J. C 59, 99 (2009), arXiv:0707.4052[hep-th]; H. Wei and R. G. Cai, Phys. Lett. B 655, 1 (2007), arXiv:0707.4526 [gr-qc]; M. Quartin, M. O. Calvao, S. E. Joras, R. R. R. Reis and I. Waga, JCAP 0805, 007 (2008), arXiv:0802.0546[astro-ph].

[10] J. Valiviita, E. Majerotto, and R. Maartens, JCAP 0807, 020 (2008), arXiv:0804.0232[astro$\mathrm{ph}$.

[11] J. H. He, B. Wang, and E. Abdalla, Phys. Lett. B 671, 139 (2009), arXiv:0807.3471[gr-qc].

[12] B. M Jackson and A. Taylor, Phys. Rev. D 79, 043526 (2009), arXiv:0901.3272[astro-ph.CO].

[13] M. B. Gavela, D. Hernandez, L. L. Honorez, O. Mena, and S. Rigolin, JCAP 0907, 034 (2009), arXiv:09011.611[astro-ph]. 
[14] P. S. Corasaniti, Phys. Rev. D 78, 083538 (2008), arXiv:0808.1646[astro-ph].

[15] H. Kodama and M. Sasaki, Prog. Theor. Phys. Suppl. 78, 1 (1984); P. K. S. Dunsby, M. Bruni and G. F. R. Ellis, Astrophys. J. 395, 54 (1992).

[16] C. P. Ma and E. Bertschinger, arXiv:astro-ph/9506072. 